\section{PSICOLOGÍA IBEROAMERICANA}

\section{Psicología lberoamericana}

ISSN: 1405-0943

psicología.iberoamericana@uia.mx

Universidad Iberoamericana, Ciudad de

México

México

Martínez-Jerez, Ana María; Morales-Carmona, Francisco

Validación de la Encuesta sobre pareja y familia en el proyecto vital de los jóvenes

Psicología Iberoamericana, vol. 21, núm. 2, julio-diciembre, 2013, pp. 60-71

Universidad Iberoamericana, Ciudad de México

Distrito Federal, México

Disponible en: http://www.redalyc.org/articulo.oa?id=133930525007

Cómo citar el artículo

Número completo

- Más información del artículo

Página de la revista en redalyc.org

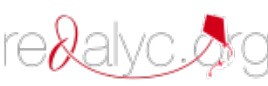

Sistema de Información Científica

Red de Revistas Científicas de América Latina, el Caribe, España y Portugal

Proyecto académico sin fines de lucro, desarrollado bajo la iniciativa de acceso abierto 


\title{
Validación de la Encuesta sobre pareja y familia en el proyecto vital de los jóvenes
}

\author{
Validation of the Couple and Family Survey in the Life Project of Young People
}

\author{
Ana María Martínez-Jerez ${ }^{1}$ \\ Universidad Autónoma de TAMAULIPAS \\ Francisco Morales-Carmona \\ instituto Nacional de Perinatolocía
}

\section{RESUMEN}

La presente encuesta, elaborada en Alemania para un proyecto internacional comparado entre seis países, sobre los proyectos de vida en jóvenes de ambos sexos, fue adaptada y validada en estudiantes de una universidad de Tamaulipas, México. Suministrada ya en español, la adaptación fue realizada por seis especialistas y evaluada en una prueba piloto con 93 alumnos y alumnas de veintiún a treinta y cinco años del último semestre de la carrera de Psicología. Se efectuó la validación de contenido por expertos y validación de constructo a través de análisis factorial y de componentes principales categóricos, con 635 alumnos y alumnas de dieciocho a treinta y seis años de seis licenciaturas universitarias. Asimismo, se midieron los índices de confiabilidad. Se concluye que el instrumento cuenta con los requerimientos psicométricos necesarios para ser utilizado en la población mexicana y que, efectivamente, mide diversos aspectos del proyecto de vida en pareja.

Palabras clave: validez de contenido, validez de constructo, análisis factorial, proyecto de vida en pareja, estudiantes universitarios.

\section{ABSTRACT}

This survey, devised in Germany for an international project comparing six countries in regards the life projects of young people of both sexes, was adapted and validated for students at a university in Tamaulipas, Mexico. The survey was carried out in Spanish and adapted by six specialists and evaluated during a pilot program involving 93 pupils aged between 21 and 35, during their final semester of the undergraduate psychology program. Experts carried out a content and construct validation using factor analysis and the analysis of the main categorical components or 635 pupils aged between 18 and 36 studying six undergraduate programs. Reliability indices were also measured. We conclude that the instrument has the necessary psychometric requirements to be applied to the Mexican population and that it is effective as a measurement of various aspects of people's life projects when living as couples.

Keywords: content validity, construct validity, factor analysis, life project for couples, university students.

\footnotetext{
${ }^{1}$ Recibido: 1 de octubre, 2013 - Aceptado: 12 de agosto, 2013

Correspondencia: Ana María Martínez Jerez. Universidad Autónoma de Tamaulipas. Blvd. Adolfo López Mateos esq. Av. Universidad s/n. Tampico, Tamps. C.P. 89138. Tel. 833241 2000, ext. 3665. anamariamj@hotmail.com

Francisco Antonio Morales Carmona. Instituto Nacional de Perinatología. Prado Sur 800, Lomas de Chapultepec, Miguel Hidalgo, C.P. 11000. México, D. F. fmoralesc@inper.mx
} 


\section{INTRODUCCIÓN}

El unirse en pareja y formar una familia son etapas que la mayoría de los seres humanos deciden vivir. Ambas conforman procesos complejos que involucran la satisfacción de múltiples necesidades biológicas, psicológicas y culturales. Entre las biológicas se mencionan el placer de la sexualidad y la continuación de la especie, que son consideradas importantes por muchas parejas (Valdez, González \& Sánchez, 2007). Las psicológicas involucran el deseo de amar y ser amados, vincularnos, expresar el afecto, etc. Las culturales, en forma de creencias, reglas y normas, determinan las maneras en que se estructura y manifiesta dicho vínculo amoroso, y pueden incluir desde la búsqueda de reconocimiento familiar, prestigio social, consenso de valores y de bienes económicos, hasta la estructura de poder en la pareja, por mencionar algunas (Gangestad, Haselton \& Buss, 2006; Pérez, Rangel, Jiménez \& Freyre, 2007; Sánz, 1999).

Esta formación de la pareja se ha validado históricamente a través del matrimonio. Sin embargo, es un hecho que en la actualidad dicho esquema ha sufrido cambios, tanto en México como en otros países. En nuestra sociedad, hoy, el ser humano ha optado por diversas formas de vivir en pareja: dentro de un matrimonio o como una unión libre; como primera o consecuente unión; con una persona del mismo o de diferente sexo; sin hijos o con hijos que pueden ser de uno de los integrantes de la pareja o bien de ambos o adoptados; con un compromiso permanente o como prueba para confirmar un compromiso y así casarse posteriormente, etc. (Fitzgibbons, 2006; Meil, 2004; Padilla \& Díaz-Loving, 2012; Romo, 2009).

Así, de manera independiente de la forma que asuma, el ser humano sigue en búsqueda de otro ser con quien llegar a consolidar un proyecto de vida en común (García \& Reyes, 2009; García, Salvador \& Guzmán, 2012). De hecho, diversos autores reportan que, tanto en México como en otras sociedades, tener un buen trabajo y formar una familia siguen siendo los dos principales objetivos vitales en las nuevas generaciones, y que el matrimonio es todavía, entre todas las opciones, la más elegida (García \& Reyes, 2009; Meil, 2004; Romo, 2008, 2009), incluso entre los hijos de padres divorciados o separados (Durán, Medina, González \& Rolón, 2007; Jones \& Nelson, 1997; Meil,
2008). Asimismo, se ha encontrado que, por lo menos en el nivel consciente, es común reportar el predominio del amor como factor fundamental para formar una pareja (Meil, 2004).

En cuanto a los hijos, Romo (2007) y otros autores (Garrido, Reyes, Torres \& Ortega, 2008) encuentran que, en nuestra cultura mexicana, las parejas tienen expectativas importantes con la llegada de éstos y que, incluso, en algunos momentos más que una elección privada pareciera ser un imperativo social. No obstante, también hay un interés en los jóvenes mexicanos actuales de tener un número reducido de hijos para proporcionarles mejor calidad de vida.

En concordancia con lo reportado por el Consejo Nacional de Población (Conapo, 2011), Romo (2007) encuentra que el número de hijos deseados oscila entre dos y tres, y que algunos de los jóvenes mexicanos que entrevistó (Romo, 2009) se plantean la posibilidad de una vida en pareja sin que exista matrimonio, y que esta relación no conllevaría, precisamente, la formación de una familia con el concepto tradicional de tener hijos, sino que podría existir la pareja sin ellos, a la cual también se le podría considerar una familia. Así, estos elementos como formar o no una pareja, casarse o no, tener hijos o no, el número de ellos (Romo, 2007) etc., conforman aspectos importantes de un proyecto de vida en pareja.

Pero, ¿qué entenderíamos por proyecto de vida o proyecto vital en pareja?, y ¿para qué sería importante conocerlo? Para Blas y Rojas (2008), las personas se trazan objetivos y metas de vida importantes que desean alcanzar en un corto, mediano o largo plazos, aunque esta planificación no se haga siempre de manera consciente; a esto llamarían proyecto de vida en general.

Aunque algunos autores consideran que es durante la adolescencia, y de la mano del proceso de autonomización, cuando los individuos comienzan a construir sus proyectos de vida (Aisenson, 1998; Climent, 2003). Coincidimos con Sánz (1999) y Guichard (1995) quienes plantean que el proyecto individual de vida es un proceso que comienza a realizarse durante la infancia, y la familia de origen ejerce una influencia sustancial (Durán et al., 2007). Sin embargo, este proceso de formación se ve determinado también por la historia personal del sujeto a lo largo de su vida, es decir, por las 
experiencias positivas o negativas a las que se haya o se esté enfrentando en el transcurrir de su existencia. Así, el proyecto de vida es un proceso continuo, que podemos medir en el momento en que se entrevista o cuestiona a la persona, pero que pudiera no ser similar ni estable a través del tiempo (Meil, 2008).

Específicamente, en cuanto al proyecto vital en pareja, Romo $(2007,2008,2009)$, quien ha trabajado sobre este constructo en universitarios mexicanos, lo define como un plan de vida pero referido a las relaciones de pareja. En consonancia con Sánz (1999) y Guichard (1995), este autor considera que el proyecto total de vida se da en una intersección entre "el espacio de la experiencia y el horizonte de la expectativa" (Romo, 2009, p. 32); es decir, entre una combinación de eventos que el individuo ha vivido en el pasado, que recuerda en el presente y que, juntos, sirven de base para cualquier serie de proyectos a futuro que pretenda realizar, así como para la toma de nuevas decisiones y acciones; es el futuro hecho presente.

Y, específicamente, explica así el proyecto de vida en pareja:

De la misma manera, si hablamos en el presente de sus relaciones de pareja, la persona retoma elementos de su historia biográfica, se sitúa en sus logros presentes y además se "proyecta" en alguna alternativa: el establecimiento de una familia y la llegada de los hijos, o por el contrario, no quiere saber nada de esto, o alguna postura intermedia (sí matrimonio pero no hijos, sí hijos aunque no haya matrimonio, etc.) (Romo, 2009, p. 32).

Para este autor, conocer el proyecto de vida en pareja de cualquier individuo requiere abordar cuatro dimensiones: a) si tiene pensado casarse o no, b) si considera tener hijos y cuántos, c) en qué momento de la vida le gustaría casarse y d) si tiene pensado que sea de por vida (Romo, 2007).

Para los fines de la presente investigación, entenderemos proyecto de vida en pareja como una serie de representaciones o visualizaciones sobre las expectativas que tiene un individuo de una futura vida en pareja, en diversas dimensiones. Estas representaciones tendrán como base una integración de la historia biográfica del individuo, su vida actual y el futuro desea- do. Operacionalmente, proyecto de vida en pareja será entendido como la configuración del total de respuestas a la Encuesta sobre pareja y familia en el proyecto vital de los jóvenes (EPF) a través de las ocho dimensiones teóricas planteadas por el mismo instrumento, las que se mencionarán más adelante.

Sobre la utilidad de medir el constructo "proyecto de vida en pareja" desde un punto de vista preventivo, diversos autores subrayan la importancia de conocer qué sucede con las parejas desde el momento en que se establece la unión, para determinar posibles factores de riesgo que precederían y asociarían con disfunción conyugal (Jones \& Nelson, 1997; Kim \& McKenry, 2002; Robles \& Kiecolt-Glaserb, 2003). Algunos de esos factores de riesgo reportados son la influencia de ambas familias de origen, decisiones sobre la educación de los hijos, religión, conciliación entre vida familiar y laboral, organización en el hogar y roles de género (González \& Espinosa, 2004), por mencionar algunos factores que, igualmente, han sido considerados como elementos de un proyecto de vida en pareja (Meil, 2004, 2005, 2008). Tener esta información y actuar en consecuencia permitiría no sólo fomentar desde el comienzo relaciones saludables y satisfactorias entre sus integrantes, sino también disminuir diversas problemáticas relacionadas con el deterioro de la vida en pareja (por mencionar dos: la violencia familiar y la separación conyugal conflictiva), así como sus consecuencias reportadas: alcoholismo, depresión, ansiedad y problemas financieros para los integrantes de la pareja y depresión, baja autoestima y dificultades académicas, entre otras, para los hijos (Jones \& Nelson, 1997; Amato \& Sobolewski, 2001).

Con el propósito de contar, en nuestro país, con un instrumento que llenara estas necesidades de detección y prevención se planteó el objetivo del presente estudio: adaptar y validar para México la EPF. Se eligió este instrumento por considerar que las múltiples dimensiones que aborda permiten medir, de forma más integral, el constructo proyecto de vida en pareja.

Existen algunos instrumentos que miden conceptos muy similares. En Estados Unidos, Jones y Nelson (1997) elaboraron una escala para medir las expectativas maritales en estudiantes solteros. Las dimensiones que mide son intimidad (sexualidad, comunicación y cercanía emocional), igualdad (familia, hijos, amigos 
y tareas del hogar) y compatibilidad (actividades recreativas conjuntas, personalidad y atractivo de la futura pareja). En México, García y Sánchez-Aragón (2005) desarrollaron una escala sobre expectativas de una relación de pareja que mide cuatro factores: a) compatibilidad-equidad, b) romance, c) ajuste afectivo e instrumental y d) relación con otros. Ésta mostró estabilidad robusta y congruencia conceptual con la escala de Jones y Nelson (1997).

En los reportes de España sobre el estudio para el que fue elaborado la EPF, Meil $(2004,2005,2008)$ menciona que el objetivo general que se persiguió fue analizar la importancia y el significado que las nuevas generaciones de jóvenes españoles atribuyen a la formación de una pareja, al matrimonio, a la constitución de una familia y a otros modos alternativos de convivencia en sus proyectos de vida, así como analizar las pautas de cambio o permanencia familiar presentes en la sociedad española (Meil, 2004, 2005). De acuerdo con los objetivos particulares mencionados por el mismo autor y a la configuración teórica de los reactivos del instrumento, los elementos que conformarían estos proyectos vitales serían: 1) deseo de unirse en pareja y formar una familia, 2) importancia de la familia de origen en el proyecto, 3) equivalencia entre matrimonio y unión libre, 4) idea de familias e hijos, 5) tareas en el hogar, organización en familia y roles de género, 6) conciliación entre vida familiar y laboral, 7) opinión sobre el divorcio y 8) expectativas en torno a pareja y matrimonio (Meil, 2005). Para el presente estudio, estos ocho elementos son considerados dimensiones que conforman el constructo proyecto de vida en pareja medidos por la EPF.

Existen algunas limitaciones importantes para esta investigación. En primer lugar, la EPF fue aplicada a una muestra no aleatoria de estudiantes de ambos sexos en una zona específica del noreste de México. Debido a la diversidad y la complejidad de nuestra cultura y estructura poblacional, su generalización a otras zonas del país debe tomarse con cautela. En segundo lugar, no se reportan datos sobre la validación original o la adaptación y validación para España de la EPF, lo que dificulta la comparación numérica con lo obtenido en México, por ejemplo, con respecto a la confiabilidad. Sin embargo, Meil $(2004,2005,2008)$ es muy claro y analítico, teóricamente, con respecto a lo que midió la
EPF en España, por lo que, en ese nivel, es posible determinar semejanzas y diferencias en los resultados de la validación en nuestro país. Así, estas limitantes pueden ser subsanadas y los autores consideramos que la EPF será de gran utilidad en nuestra población, pues se contará con un instrumento validado y adaptado que permitirá evaluar, de forma más integral, los proyectos de vida y sus implicaciones para nuestros jóvenes.

\section{MÉTODO}

\section{Participantes}

Para la prueba piloto fueron seleccionados, de manera intencional (López \& Juárez, 2004), 93 jóvenes de ambos sexos (20 varones y 73 mujeres), de veintiún a treinta y cinco años, estudiantes del último semestre de la Licenciatura en Psicología de una universidad del estado de Tamaulipas, México. La aplicación final se llevó a cabo con 635 alumnos y alumnas (208 y 427, respectivamente), estudiantes de seis diferentes licenciaturas de la misma universidad: Turismo, Derecho, Psicología, Inglés, Comunicación y Economía, seleccionados de manera no probabilística por accidente (Kerlinger \& Lee, 2008) y con edades entre dieciocho y treinta y seis años.

\section{Instrumento}

La EPF fue diseñada por el Seminario Interdisciplinar de Familia, de la Universidad de Oldenburg, en Alemania, para un proyecto internacional comparado entre seis países sobre los proyectos de vida de jóvenes universitarios de ambos sexos. Además de España y Alemania, los otros países participantes fueron Polonia, Lituania, Corea y Chile (Busch \& Scholz, 2006; Meil, 2005, 2008). Gerardo Meil, profesor del Departamento de Sociología de la Universidad Autónoma de Madrid, y quien encabeza el equipo español encargado de participar en dicho proyecto, facilitó y autorizó la adaptación y la validación de dicha encuesta para México, sin existir relación alguna de este país con el proyecto internacional citado.

Así, la encuesta que se nos proporcionó originalmente fue la utilizada en España y, de acuerdo con los resultados y los análisis reportados por Meil (2004, 2005, 2008), podríamos clasificar a la EPF como un cuestionario de cotejo (Jurado, 2002); es decir, un ins- 
trumento para estimar índices de ocurrencia, tipos, formas y características diversas de un fenómeno, cuyos resultados se presentan en porcentajes o promedios de cada reactivo y con un análisis y discusión teórica del significado de cada uno. Esto quiere decir, por tanto, que en la EPF no se obtiene una puntuación global o por componentes o escalas.

La EPF es un instrumento estructuralmente complejo. Aunque la numeración inicial de la encuesta es de 54 reactivos, consta de 61 debido a que algunos, por razones no especificadas, se subdividen en dos y hasta en tres incisos, si bien cada uno pregunta cosas diferentes. Por ejemplo, el reactivo tres cuestiona: ¿por cuál de los siguientes motivos te casarías?, y el siguiente: ¿a qué edad te gustaría casarte? se numera como 3a). También, la mayoría de los reactivos está conformada por diversos subreactivos. Por ejemplo, el reactivo siete "objetivos en la vida" consta de 14 opciones o subreactivos como: a) tener éxito en mi carrera profesional/trabajo, b) formar mi propia familia, etc. Así, el total de reactivos y subreactivos que la componen es de 311. Igualmente, la EPF está conformada por tres tipos de respuesta: 14 de escala tipo Likert de tres a seis opciones, ocho preguntas abiertas numéricas (¿a qué edad te gustaría casarte?) y 39 reactivos categóricos (de nivel ordinal y nominal). Aunque incluye reactivos demográficos (edad, sexo, estado civil), éstos no están colocados al inicio como ocurre por lo general, sino que forman parte del resto y se ubican casi al final de la encuesta. Se contesta en un tiempo aproximado de cuarenta y cinco minutos.

\section{Procedimiento}

Para el proceso de adaptación de la encuesta a México se tomaron como guía los lineamientos propuestos por Geisinger (1994), así como los procedimientos de adaptación y validación del Inventario de Depresión de Beck, realizado por Jurado y colaboradores en 1998. El procedimiento se dividió en dos fases: a) la adaptación y la validación de contenido, y b) la validación de constructo y la confiabilidad. Para la adaptación, el primer paso fue realizado por los autores de la presente investigación, efectuando modificaciones a palabras, frases y modismos españoles no utilizados en la cultura mexicana. Por ejemplo, se cambiaron las palabras "piso" por casa o departamento y "uniones de hecho" por unión libre. Adicionalmente, se realizaron ajustes en la ubicación y el número de reactivos y subreactivos: a) se reubicaron al inicio los reactivos demográficos de la encuesta original, b) se agregaron otros demográficos (carrera, semestre, grupo) y c), como ya se comentó, se modificó y se dio continuidad a la numeración original, con 61 reactivos en lugar de 54.

De aquí surgió una primera versión de la EPF, evaluada por seis expertos en terapia familiar y con amplia experiencia en psicometría, a fin de que fuera revisada tanto en forma como en contenido (para evaluar la validez de contenido). Se les solicitó que evaluaran: 1) si la forma en que se encontraban redactados los reactivos, así como el lenguaje utilizado eran claros y comprensibles para población mexicana, y 2) si el instrumento estaba midiendo efectivamente proyecto de vida en pareja. Con respecto a la adaptación del lenguaje hubo comentarios y sugerencias de cambios importantes. Con base en estos cambios y recomendaciones de los expertos se modificó el instrumento, de donde surgió una segunda versión. Los resultados de la validez de contenido se comentarán más adelante.

Esta segunda versión fue sometida a una prueba piloto con 93 jóvenes de ambos sexos, como ya se comentó, quienes fueron seleccionados de manera intencionada (López \& Juárez, 2004) por contar con conocimientos psicométricos para lograr, de mejor forma, el objetivo propuesto de esta aplicación, de manera escrita y realizada en los salones de clase; éste consistió en confirmar que todos los reactivos fueran bien comprendidos y culturalmente adecuados para jóvenes, así como verificar aspectos prácticos como el tiempo de aplicación y la claridad en las instrucciones. Una de las sugerencias importantes fue no considerar cuatro subreactivos que generaban cierta confusión, pues preguntaban "otros motivos" de manera cerrada, sin posibilidad de que el participante brindara mayor información. Sobre la base de los comentarios y las modificaciones sugeridas en esta prueba piloto se elaboró una tercera versión de la encuesta, la cual fue sometida a la segunda fase del estudio: la aplicación definitiva para realizar los procedimientos estadísticos de validez de constructo y confiabilidad.

Esta tercera versión quedó constituida por 63 reactivos y 307 subreactivos: seis de datos demográficos, 14 de escala tipo Likert, 36 reactivos categóricos y siete 
de respuestas abiertas numéricas. El cuestionario se diseñó para ser contestado en línea, y se aplicó de manera voluntaria, anónima y confidencial, en el centro de cómputo de la misma universidad, a 635 alumnos y alumnas elegidos de manera accidental, estudiantes de seis diferentes licenciaturas y en un tiempo aproximado de cuarenta y cinco minutos, como ya se comentó.

Debido a la estructura de la encuesta, los análisis estadísticos utilizados para medir la validez del constructo y observar la estructura subyacente de la EPF fueron dos: 1) análisis factorial exploratorio con método de extracción por componentes principales y método de rotación de normalización varimax con Kaiser para los reactivos tipo Likert y numéricos abiertos, y 2) análisis de componentes principales categóricos (CAPTCA) en los reactivos de nivel categórico. Para conformar los factores y los componentes se consideraron los siguientes criterios: a) valor propio superior a uno, b) carga factorial o saturación de componentes mayor o igual a 0.40, c) congruencia conceptual entre los reactivos de un componente o factor y d) más de dos reactivos agrupados en cada factor o componente. La confiabilidad fue medida a través del alfa de Cronbach. Todos los análisis se realizaron con el sistema spss 17.

\section{RESULTADOS}

Como se mencionó anteriormente, la EPF fue evaluada por expertos para asegurar la validez del contenido. Por unanimidad, los jueces determinaron que la encuesta, en efecto, medía el constructo proyecto de vida en pareja. Se hicieron ajustes generales según las recomendaciones dadas en dicho proceso.

Geisinger (1994) menciona que la mejor forma de validar un instrumento de una cultura a otra es la validación de constructo. Así, este paso fue fundamental para el objetivo propuesto.

\section{Resultados de los reactivos con nivel de medición escalar}

La medida de adecuación muestral KMO (0.796) y la prueba de esfericidad de Bartlett (14265.7, p = 0.000) fueron significativas para estos reactivos, lo que indica el ajuste de la matriz de correlaciones para llevar a cabo el análisis factorial (Kerlinger \& Lee, 2008). A partir del análisis factorial exploratorio, de los 21 reac- tivos escalares validados (14 tipo Likert y siete abiertos numéricos, 121 subreactivos), se eliminaron diez completos (los siete reactivos abiertos numéricos y tres tipo Likert, 46 subreactivos), por bajas cargas factoriales. Ejemplos de ítems eliminados: número de hermanos y hermanas ( 48 y 49), ¿te consideras una persona religiosa? (52), vivo solo/sin pareja (63.4).

Finalmente, se obtuvo una estructura de ocho factores que en conjunto explican $47.12 \%$ de la variabilidad. Éstos fueron: F1) organización de las tareas del hogar, F2) equivalencia entre matrimonio y unión libre, F3) condiciones para iniciar y mantener una pareja, F4) razones para el divorcio, F5) objetivos personales complementarios, F6) idea de matrimonio e hijos, F7) importancia de la familia de origen y F8) expectativas básicas en torno a pareja y matrimonio. Se obtuvo una confiabilidad total de 0.843 . Así, la encuesta quedó conformada por 11 reactivos y 75 subreactivos escalares.

Algunos ítems que constituyen cada factor, sus cargas factoriales y las confiabilidades por factor se presentan en la tabla 1. 
Tabla 1. Estructura, cargas factoriales y confiabilidades de la EPF. Reactivos escalares

\begin{tabular}{|c|c|c|c|c|c|c|c|c|}
\hline Reactivos & F1 & F2 & F3 & F4 & F5 & F6 & F7 & F8 \\
\hline 62.1 Preparar mi desayuno o comida & 0.545 & & & & & & & \\
\hline 62.3 Poner o recoger la mesa & 0.725 & & & & & & & \\
\hline 62.5 Barrer y/o trapear la cocina & 0.773 & & & & & & & \\
\hline 62.10 Lavar la ropa de todos & 0.609 & & & & & & & \\
\hline 13.1 Fidelidad mutua en el amor & & 0.765 & & & & & & \\
\hline 13.4 Respeto y tolerancia mutua & & 0.796 & & & & & & \\
\hline 13.6 Que ambos quieran tener niños & & 0.569 & & & & & & \\
\hline 13.8 Estar de acuerdo en temas de dinero & & 0.696 & & & & & & \\
\hline 12.9 Tener intereses comunes & & & 0.521 & & & & & \\
\hline 12.10 Tener amigos comunes & & & 0.550 & & & & & \\
\hline 12.15 Tener creencias religiosas similares & & & 0.557 & & & & & \\
\hline 12.16 Tener los mismos orígenes sociales & & & 0.683 & & & & & \\
\hline 38.1 En caso de malos tratos & & & & 0.674 & & & & \\
\hline 38.2 En caso de infidelidad sexual & & & & 0.627 & & & & \\
\hline 38.4 Cuando ha desaparecido el amor & & & & 0.714 & & & & \\
\hline 38.8 Por motivos económicos & & & & 0.488 & & & & \\
\hline 7.7 Ganar mucho dinero & & & & & 0.410 & & & \\
\hline 63.7 Tengo muchos amigos & & & & & 0.639 & & & \\
\hline 63.8 He vivido, he viajado y he conocido mundo & & & & & 0.767 & & & \\
\hline 63.10 Soy dueño de mi casa o departamento & & & & & 0.722 & & & \\
\hline 32.1 Si se quiere tener hijos es mejor casarse & & & & & & 0.460 & & \\
\hline $\begin{array}{l}32.3 \text { El casarse da más seguridad que la pareja } \\
\text { sin casarse }\end{array}$ & & & & & & 0.528 & & \\
\hline 63.2 Estoy casado & & & & & & 0.746 & & \\
\hline 63.3 Tengo hijos & & & & & & 0.713 & & \\
\hline $24 \mathrm{Mi}$ familia de origen es un modelo a seguir & & & & & & & 0.656 & \\
\hline 45.1 Relaciones con la madre & & & & & & & 0.580 & \\
\hline 45.2 Relaciones con el padre & & & & & & & 0.608 & \\
\hline 46.2 Grado de unión y apoyo & & & & & & & 0.659 & \\
\hline 7.6 Formar mi propia familia & & & & & & & & 0.559 \\
\hline 7.14 Estar a gusto con la familia que forme & & & & & & & & 0.632 \\
\hline 12.1 Fidelidad mutua en el amor & & & & & & & & 0.623 \\
\hline 12.5 Tener relaciones sexuales satisfactorias & & & & & & & & 0.462 \\
\hline Varianza por factor & 10.50 & 10.00 & 6.76 & 4.95 & 4.55 & 4.05 & 3.32 & 2.96 \\
\hline Varianza total & & & & & & & & 47.12 \\
\hline Confiabilidad por factor & 0.924 & 0.912 & 0.793 & 0.787 & 0.737 & 0.750 & 0.780 & 0.678 \\
\hline \multicolumn{8}{|l|}{ Confiabilidad total } & 0.843 \\
\hline
\end{tabular}




\section{Resultados de los reactivos con nivel de medición nominal}

De los 36 reactivos categóricos (181 subreactivos) que constituyen la EPF, fueron eliminados 12 (133 subreactivos) después de la aplicación. A partir de una revisión preliminar de la base de datos se encontraron inconsistencias importantes en la forma en que fueron contestados estos reactivos, lo que no permitía un adecuado procesamiento estadístico: $9,11,17,18,19$, $20,42,44,47,56,57$ y 58 . Éstos miden razones para tener o no tener hijos y para casarse o no casarse, cómo conjuntar trabajo e hijos, relación e información sobre los padres del participante, grado de estudios, trabajo y profesión.

A partir del análisis CATPCA se eliminaron 11 reactivos más (16 subreactivos) por bajas cargas en las saturaciones de los componentes. Algunos de los eliminados en esta fase fueron: ¿en qué situación te encuentras (trabajas, estudias, ambos)? (50), ¿cómo te consideras en materia religiosa? (51), ¿tienes pareja? (53) y situación legal de tus padres (59).

Finalmente, el análisis arrojó una estructura subyacente de siete componentes que explican $30.29 \%$ de la varianza: C1) organización de las tareas del hogar y roles de género, C2) apoyo de personas significativas en problemáticas diversas, C3) opinión sobre derechos homosexuales, C4) ideas sobre matrimonio, hijos y vida familiar y laboral, C5) importancia de los hijos en la felicidad de los padres, C6) opinión sobre madres y padres solteros y C7) equivalencia entre matrimonio y unión libre. La confiabilidad total obtenida fue de 0.981 . La encuesta quedó conformada, así, por 13 reactivos y 32 subreactivos categóricos.

Algunos ítems que conforman cada componente, sus saturaciones y confiabilidades por componentes se presentan en la tabla 2.

Tabla 2. Estructura por componentes, saturaciones y confiabilidades de la EPF. Reactivos categóricos

\begin{tabular}{|c|c|c|c|c|c|c|c|}
\hline Reactivos & C1 & $\mathrm{C} 2$ & C3 & $\mathrm{C} 4$ & C5 & $\mathrm{C} 6$ & $\mathrm{C7}$ \\
\hline 61.3 Arreglar cosas descompuestas & 0.634 & & & & & & \\
\hline 61.4 Cuidar del cónyuge/hijos enfermos & 0.758 & & & & & & \\
\hline 61.7 Cocinar & 0.779 & & & & & & \\
\hline 61.8 Cuidar de las plantas y/o animales & 0.700 & & & & & & \\
\hline 43.5 Cuestiones de fe & & 0.500 & & & & & \\
\hline 43.6 Problemas con amigos & & 0.517 & & & & & \\
\hline 43.7 Problemas de amor & & 0.559 & & & & & \\
\hline 43.8 Problemas familiares & & 0.463 & & & & & \\
\hline 28.7 Dos hombres homosexuales con al menos un hijo & & & 0.682 & & & & \\
\hline 28.8 Dos mujeres homosexuales con al menos un hijo & & & 0.674 & & & & \\
\hline 35 Derechos de parejas homosexuales & & & 0.530 & & & & \\
\hline 8 Estado civil en el futuro & & & & 0.631 & & & \\
\hline 14 Quiero tener hijos & & & & 0.577 & & & \\
\hline 29 Matrimonio, institución pasada de moda & & & & 0.518 & & & \\
\hline 39 Roles de hombres y mujeres en casa & & & & 0.463 & & & \\
\hline 26 Mujer feliz con hijos o sin hijos & & & & & 0.622 & & \\
\hline 27 Hombre feliz con hijos o sin hijos & & & & & 0.618 & & \\
\hline 22. Un niño criarse sólo con su papá o mamá & & & & & & 0.742 & \\
\hline 28.5 Una madre sola con al menos un hijo & & & & & & 0.592 & \\
\hline 28.6 Un padre solo con al menos un hijo & & & & & & 0.592 & \\
\hline 33.1 El matrimonio beneficia más a & & & & & & & 0.666 \\
\hline 33.2 Vivir juntos sin casarse beneficia más a & & & & & & & 0.647 \\
\hline Varianza total & & & & & & & 30.29 \\
\hline Confiabilidad por componente & 0.845 & 0.760 & 0.709 & 0.615 & 0.515 & 0.436 & 0.384 \\
\hline \multicolumn{7}{|l|}{ Confiabilidad total } & 0.981 \\
\hline
\end{tabular}


Así, el cuestionario final quedó conformado por treinta reactivos: seis de datos demográficos, 11 (75 subreactivos) con nivel de medición escalar y 13 (32 subreactivos) categóricos.

\section{DISCUSIÓN}

Iniciaremos la discusión planteando la siguiente pregunta de Geisinger (1994), importante en cualquier proceso de adaptación y validación: ¿las puntuaciones del nuevo instrumento significan lo mismo que en la cultura y en el lenguaje del instrumento original? Por sus características particulares, en la EPF se puede responder que sí y que no. Al ser un instrumento que no posee una puntuación única o por componentes o escalas, y de que originalmente su análisis se basa en la interpretación teórica de cada reactivo, hay un sentido de diferenciación según el contexto y la cultura en donde se use. De hecho, como ya se mencionó, fue creada para un estudio comparativo entre países. En este sentido, las puntuaciones de la EPF tampoco significarán lo mismo al ser usada para diferentes propósitos. Por ejemplo, el planteado por Busch y Scholz (2006) y por Meil $(2004,2005,2008)$ para el uso de la EPF no es el mismo que el que se pretende en la presente investigación. El nuestro es predictivo, el de Meil es hacer un análisis de una situación social actual en España, y el de Busch y Scholz fue realizar un análisis comparativo entre diversos países sobre la forma en que los jóvenes perciben, de manera tradicional o progresista, la familia y el matrimonio. Por supuesto, es posible que algún investigador decida utilizarlo en México con estos mismos u otros objetivos.

Sin embargo, más allá de estas particularidades, éste y cualquier instrumento deben tener cierta homogeneidad y consistencia teórica que permita asegurar que, en diferentes contextos y culturas, mide un mismo constructo y, a la vez, tener la seguridad de que las formas en que lo mide son congruentes con las características de la nueva población. Evaluar esto en la EPF fue el objetivo principal del presente estudio.

En primer lugar, aunque Meil (2004, 2005, 2008) no precisa a qué llama proyecto vital de pareja y familia, los resultados con expertos de la validación de contenido de la EPF nos permiten aseverar que, efectivamente, mide dimensiones de dicho constructo y los resultados de las confiabilidades nos dicen que existe una adecuada consistencia en el grado en que los ítems se correlacionan entre ellos para medirlo.

En segundo lugar, los resultados de la validez de constructo permiten afirmar que hay una concordancia entre la estructura de factores y componentes obtenidos con los constructos teóricos propuestos por diferentes autores (Durán et al., 2007; González \& Espinosa, 2004; Romo, 2007). Igualmente, de las ocho dimensiones planteadas por Meil (2005) como constituyentes de la EPF, todas están representadas en los resultados obtenidos, cuatro de ellas en ambos niveles de medición: importancia de la familia de origen, equivalencia entre matrimonio y unión libre, ideas sobre familia e hijos y organización de las tareas del hogar. En los reactivos escalares se representaron el deseo de unirse y formar una familia, razones para el divorcio y expectativas básicas en torno a pareja y matrimonio. Aparecieron dos factores nuevos: condiciones para iniciar y mantener una pareja, y objetivos personales complementarios. En los reactivos categóricos sólo se representó la dimensión original de conciliación entre vida familiar y laboral, pero dentro del componente ideas sobre familia e hijos, no como uno separado como lo plantea Meil (2005). En estos reactivos categóricos se encontraron tres nuevos componentes: importancia de los hijos en la felicidad de los padres, opinión sobre madres y padres solteros, y postura ante los derechos homosexuales. En total, para la población de jóvenes solteros mexicanos la EPF mide 11 dimensiones del proyecto de vida en pareja.

Este agregado de dimensiones en relación con el instrumento original español puede deberse a una adaptación aceptable y, por lo tanto, a la expresión, en el instrumento, de las diferencias culturales de jóvenes mexicanos de una zona específica del norte del país, con respecto a los españoles, en cuanto a sus proyectos de vida en pareja. Así, nuestros jóvenes comparten, en paralelo, diversas dimensiones del constructo pero, al parecer, dan una importancia diferencial a otras, como a las condiciones para iniciar y mantener una pareja, a los hijos, para su propia felicidad, a los objetivos personales más allá de los familiares, a la posibilidad de vivir como padres o madres solteros y al tema social de los derechos homosexuales. Esto muestra que los reactivos y los subreactivos que se eliminaron durante el proceso de validación no tuvieron mayor repercu- 
sión en la medición del constructo en nuestro país, pues o no medían dimensiones relevantes al proyecto de vida en pareja para nuestra población o la dimensión que representaban dichos ítems ya estaba medida en otros reactivos y subreactivos. Pero también, de acuerdo con esta reconformación, al parecer los jóvenes mexicanos consideran la conciliación entre vida familiar y laboral como un tema tan importante que lo integran en las ideas generales que tienen sobre matrimonio, familia e hijos. Igualmente, el deseo de unirse y formar una pareja lo vinculan de manera directa a las expectativas básicas que pudieran tener en torno a la pareja y a la familia, es decir, pareciera ser que, en general, nuestros jóvenes del norte del país dan por hecho que, en algún momento de su vida, desearán unirse en pareja y no hacen diferencia entre esto y las expectativas que tienen en cuanto a ella.

Ello muestra que la versión de la EPF para México es adecuada y relevante para nuestra población y puede brindarnos datos válidos y confiables sobre los proyectos de vida en pareja de jóvenes universitarios. Sin embargo, es posible mejorarla y compartimos las siguientes recomendaciones: a) es importante realizar validaciones en diferentes zonas geográficas del país con el fin de garantizar la generalización e incrementar la posibilidad de recuperar la diversidad de la población, b) delimitar, para mayor especificidad en el constructo, la población de estudio con jóvenes solteros, sin hijos y que no hayan tenido experiencia de vida en común con una pareja, c) puesto que la EPF está enfocada a parejas heterosexuales se sugiere validar y adaptar también para que pueda ser utilizada en pare- jas homosexuales, d) verificar las instrucciones de los reactivos que tuvieron que eliminarse por inconsistencias en sus respuestas, pues es probable que haya habido errores en su adaptación, e) considerar reformular los contenidos de cinco reactivos abiertos numéricos eliminados por bajas cargas factoriales en reactivos de otro tipo, por ejemplo categóricos, con el objetivo de lograr mayor homogeneidad en la forma de calificar, sean validados nuevamente y, quizá, puedan ser reincluidos. Aunque las dimensiones hijos y matrimonio ya están consideradas en otros reactivos, tres de estos reactivos abiertos numéricos, como 10) la edad deseada para casarse, 15) el número de hijos deseados y 16) la edad deseada para tener el primer hijo, son indicadores específicos y fundamentales para un proyecto de vida en pareja (Romo, 2007); por eso, creemos relevante que se considere la posibilidad de reincluirlos. Los restantes dos reactivos eliminados que se sugiere que sean reformulados abordan un tema no mencionado por Meil $(2004,2005,2008)$ entre los objetivos de la EPF, y que no es analizado teóricamente en los artículos sobre su uso y resultados, pero cuya inclusión consideramos de la mayor importancia para evaluar un proyecto de vida en pareja: haber vivido o ejercido violencia en el noviazgo (reactivos 54 y 55). Se sabe que este tipo de violencia es un factor de riesgo y un detonante para continuar viviendo violencia al establecerse la pareja (INEGI, 2008). Así, la EPF es un instrumento que evalúa múltiples dimensiones de un proyecto de vida en pareja, por lo que incrementa las posibilidades de una medición más integral y completa del constructo. • 


\section{REFERENCIAS}

Aisenson, D. (1998). La transición de los jóvenes que finalizan la escuela secundaria y los adultos mayores jubilados: proyectos y recursos personales. VI Anuario de Investigaciones de la Facultad de Psicología. Buenos Aires: Universidad de Buenos Aires.

Amato, P. R. \& Sobolewski, J. M. (2001). The effects of divorce and marital discord on adult children's psychological well-being. American Sociological Review, 66 (6), 900-921.

Blas, Y. A. \& Rojas, A. M. (2008). (Una teoría del desarrollo)... para construir el futuro. Biblioteca virtual de Derecho, Economía y Ciencias Sociales. Recuperado de www.eumed.net/libros/2008a/360/indice.htm

Busch, F. W. \& Scholz, W. (2006). Familienvorstellungen zwischen fortschrittlichkeit und beharrung: ergebnisse einer empirischen untersuchung von ehe- und familienvorstellungen jugendlicher im internationalen vergleich. Würzburg: Ergon Verlag.

Climent, G. (2003). La maternidad adolescente, una expresión de la cuestión social. El interjuego entre la exclusión social, la construcción de la subjetividad y las políticas públicas. Revista Argentina de Sociología, 1 (1), 77-93.

Consejo Nacional de Población (Conapo) (2011). La situación demográfica de México 2011. México: Conapo.

Durán, A. S., Medina, A., González, N. I. \& Rolón, I. (2007). Relación entre la experiencia de separación parental y la construcción de un proyecto de vida del joven y la joven universitarios. Universitas Psychologica, 6, 713-725.

Fitzgibbons, S. E. (2006). Are men or women more reluctant to marry in couples sharing a non-marital birth? Gender Issues, 23 (I), 20-43.

Gangestad, S. W., Haselton, M. G. \& Buss, D. (2006). Evolutionary foundations of cultural variation: evoked culture and mate preferences. Psychological Inquiry, 17 (2), 75-95.

García, F. A. \& Sánchez-Aragón, R. (2005). Lo que pienso de mi relación: efectos de las expectativas y fases de la pasión. Memorias del III Congreso Mexicano de Relaciones Personales. Acapulco.

García, M. M. \& Reyes, L. I. (2009). Actitudes hacia el matrimonio y el divorcio: diferencias por sexo, residencia y estatus de pareja. Psicología Iberoamericana, 17 (1), 48-56.
García, M. M., Salvador, G. A. \& Guzmán, S. R. (2012). Actitudes hacia la transformación de la vida en pareja: soltería, matrimonio y unión libre. Psicología Iberoamericana, 20 (2), 16-25.

Garrido, G. A., Reyes, L. A., Torres, V. L. \& Ortega, S. P. (2008). Importancia de las expectativas de pareja en la dinámica familiar. Enseñanza e Investigación en Psicología, 13, 231-238.

Geisinger, K. F. (1994). Cross-cultural normative assessment: translation and adaptation issues influencing the normative interpretation of assessment instruments. Psychological Assessments, 6, 304-312.

González, M. S. \& Espinosa, S. M. (2004). Parejas jóvenes y divorcio. Revista electrónica de Psicología Iztacala, 7(1), 16-32. Recuperado de www.iztacala.unam.mx/ carreras/psicologia/psiclin/principal.html

Guichard, J. (1995). La escuela y las representaciones de futuro de los adolescentes. Barcelona: Laertes.

Instituto Nacional de Estadística, Geografía e Informática (INEGI) (2008). Referencias conceptuales de la Encuesta Nacional sobre Violencia en el Noviazgo 2007. México: INEGI.

Jones, G. \& Nelson, E. S. (1997). Expectations of marriage among college students from intact and non-intact homes. Journal of Divorce and Remarriage, 26, 171-189.

Jurado, S. (2002). Génesis, sintomatología y tratamiento del trastorno de pánico en población del D.F. (Tesis de doctorado). Universidad Nacional Autónoma de México, México.

Jurado, S., Villegas, M. E., Méndez, L., Rodríguez, F., Loperena, V. \& Varela, R. (1998). La estandarización del inventario de depresión de Beck para los residentes de la Cd. de México. Salud Mental, 21(3), 26-31.

Kerlinger, F.N. \& Lee, H.B. (2008). Investigación del comportamiento. Métodos de investigación en ciencias sociales. México: McGraw-Hill.

Kim, H. K. \& McKenry, P. C. (2002). The relationship between marriage and psychological well-being. A longitudinal analysis. Journal of Family Issues, 23(8), 885-911.

López, L. E. \& Juárez, G. F. (2004). Apuntes de métodos y técnicas de investigación en psicología social. México: Instituto Nacional de Psiquiatría Ramón de la Fuente.

Meil, L. G. (2004). La pareja en los proyectos vitales de las nuevas generaciones: deseos y realidades. Revista de Estudios de Juventud, 67, 39-55. 
Meil, L. G. (2005). Pareja y familia en el horizonte vital de las nuevas generaciones. Recuperado de www.uam. es/personal_pdi/economicas/gmeil/estadisticas. html

Meil, L. G. (2008). Pareja y familia en los proyectos vitales de las nuevas generaciones. En Meil, G. \& Torres, C. (Eds.), Sociología y realidad social. Libro homenaje a Miguel Beltrán Villalva (pp. 1195-1224). Madrid: Centro de Investigaciones Sociológicas.

Padilla, G. N. \& Díaz-Loving, R. (2012). El impacto de la cultura y la familia en la elección de pareja: diferencias entre hombres y mujeres. Psicología Iberoamericana, 20(1), 9-17.

Pérez, C. M., Rangel, P. M., Jiménez, G. J. \& Freyre, G. J. (2007). Revisión teórica de los factores que influyen en la formación de la pareja heterosexual. Psiquis (México), 16(2), 42-52.

Robles, T. F. \& Kiecolt-Glaserb, J. K. (2003). The physiology of marriage: pathways to health. Physiology and Behavior, 79(3), 409-416.
Romo, M. J. (2007). Universitarios de Aguascalientes y sus proyectos de vida en pareja. En memorias electrónicas (CD-ROM) de los Congresos Nacionales de Investigación Educativa. IX Congreso Nacional de Investigación Educativa. Mérida, Yucatán: Consejo Mexicano de Investigación Educativa, A.C.

Romo, M. J. (2008). Estudiantes universitarios y sus relaciones de pareja. Revista Mexicana de Investigación Educativa, 13, 801-823.

Romo, M. J. (2009). Jóvenes universitarios de Aguascalientes. Procesos identitarios y reflexividad moderna. (Disertación doctoral). Centro de Investigación y Estudios Avanzados del IPN-Departamento de Investigaciones Educativas, México.

Sánz, F. (1999). Los vínculos amorosos. Archivos Hispanoamericanos de Sexología, V, 7-15.

Valdez, M. J., González A. L. F. \& Sánchez, T. L. (2007). Características de personalidad percibidas en los padres y la pareja permanente: un estudio correlacional. Enseñanza e investigación en psicología, 12, 147-156. 